\title{
Nucleon-anti-nucleon intruder state of Dirac equation for nucleon in deep scalar potential well
}

\author{
T.T.S. Kuod1, T.K. Kuo ${ }^{b}$, E. Osnes ${ }^{c}$, S. Shu ${ }^{a, d}$ \\ ${ }^{a}$ Department of Physics and Astronomy, Stony Brook University, \\ Stony Brook, NY 11794, USA \\ ${ }^{b}$ Physics Department, Purdue University, W. Lafayette, IN 47907, USA \\ ${ }^{c}$ Institute of Physics, University of Oslo, NO 0316 Oslo, Norway \\ ${ }^{d}$ Department of Physics and Electronic Science, Hubei Univ., \\ Wuhan 430062, China
}

\begin{abstract}
We solve the Dirac radial equation for a nucleon in a scalar WoodsSaxon potential well of depth $V_{0}$ and radius $r_{0}$. A sequence of values for the depth and radius are considered. For shallow potentials with $-1000 \mathrm{MeV} \lesssim V_{0}<0$ the wave functions for the positive-energy states $\Psi_{+}(r)$ are dominated by their nucleon component $g(r)$. But for deeper potentials with $V_{0} \lesssim-1500 \mathrm{MeV}$ the $\Psi_{+}(r)$ s begin to have dominant anti-nucleon component $f(r)$. In particular, a special intruder state enters with wave function $\Psi_{1 / 2}(r)$ and energy $E_{1 / 2}$. We have considered several $r_{0}$ values between 2 and $8 \mathrm{fm}$. For $V_{0} \lesssim-2000 \mathrm{MeV}$ and the above $r_{0}$ values, $\Psi_{1 / 2}$ is the only bound positive-energy state and has its $g(r)$ closely equal to $-f(r)$, both having a narrow wave-packet shape centered around $r_{0}$. The $E_{1 / 2}$ of this state is practically independent of $V_{0}$ for the above $V_{0}$ range and obeys closely the relation $E_{1 / 2}=\frac{\hbar c}{r_{0}}$.
\end{abstract}

\section{Introduction}

Intruder states have played an important role in nuclear structure physics. Two of us (TTSK and EO) first learned of this subject from Gerry Brown when we were his postdocs at respectively Princeton and Nordita some long time ago. Gerry has taught, inspired and helped both of us a great deal; we are deeply grateful to him and remember well the pleasant time we had with him.

The nuclear shell model is a most succesful model for nuclear structure. A desirable feature is its large energy gap between major shells. For example, the large energy gap between the $1 s 0 d$ and $0 p$ shells allows us to treat the nucleus ${ }^{18} \mathrm{O}$ using a small $2 p 0 h$ model space, namely treating it

\footnotetext{
1 thomas.kuo@stonybrook.edu
} 
simply as two $0 s 1 d$ valence nucleons outside a closed ${ }^{16} \mathrm{O}$ core. Pioneered by Gerry, a large number of shell-model studies of nuclei using realistic nucleon-nucleon interactions have been carried out 18 The importance of intruder states in model-space effective interactions has been investigated by Schucan and Weidenmueller and others 9

In Fig. 1 we display the results of a typical calculation of ${ }^{18} O{ }^{7}$ In this calculation, a low-momentum interaction $V_{\text {lowk }}$ is employed, and the shell-model effective interaction has been calculated using three effective interaction methods denoted by LS, KK and $\mathrm{EKKO}^{7}$ in the figure. As seen, calculations give only two $2^{+}$states while experiments have three. The missing one is an 'intruder' state whose wave function is mainly outside the $2 p 0 h$ space which is the model space used for such calculation. The energies of the unperturbed $4 p 2 h$ states are much higher than the $2 p 0 h$ ones. But when the interaction is strong, some $4 p 2 h$ states are pushed down, forming an intruder $2^{+}$state whose energy is as low as those states which are dominated by the $2 p 0 h$ components. The presence of the intuder state is an indication that the Fermi sea $\left({ }^{16} \mathrm{O}\right.$-core) is no longer closed when the interaction is strong.

In the present short contribution dedicated to the memory of Gerry, we shall study the Dirac equation for a nucleon in a strong scalar potential well and discuss its possible intruder states. For a free nucleon, the negativeenergy Dirac sea is closed. But when it is subject to a strong external field, it may be no longer closed and in anlogy to the nuclear case described above, give rise to nucleon-anti-nucleon intruder states.

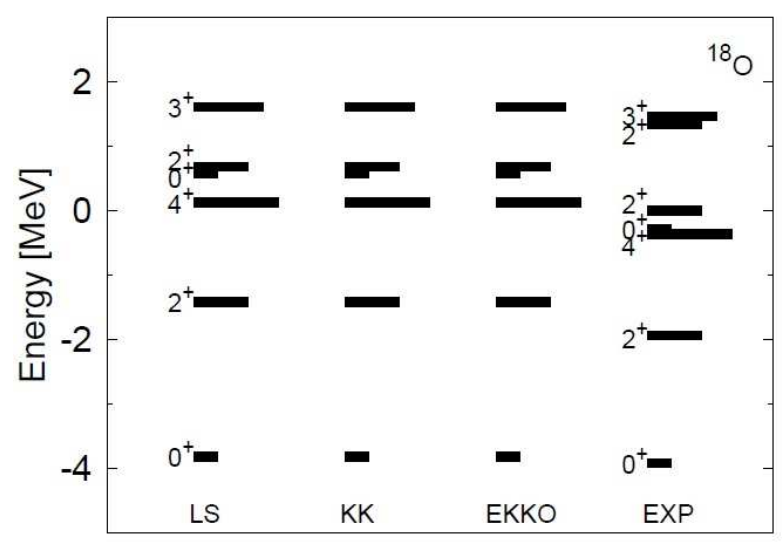

Fig. 1. Intruder states in low energy spectrum of ${ }^{18} \mathrm{O}$ 
Before discussing our present work, we would like to mention briefly Gerry's style of physics; he was fond of and successful in using simple models to bring out the simple physics of complicated problems. He often said 'This is too complicated; I can not check it with my 5-dollar calculator'. The Brown-Bolsterli schematic model for nuclear giant dipole resonance is a well known example. 11 The second-order core polarization diagram for nuclear effective interaction is another example, and has played an essential role in microscopic nuclear structure studies ${ }^{1 / 8}$ How would the properties of hadrons change with their surrounding medium is a quite complicated problem. But the Brown-Rho scaling $\frac{12 \mid 13}{1}$ provides a remarkably simple answer, namely $m^{*} / m=1-C n / n_{0}$ where $m^{*}$ and $m$ are respectively the in-medium and in-vacuum hadron mass. The medium density is $n$ and the nuclear matter saturation density $n_{0}$. For low densities, $C \simeq 0.2$. Extensive applications of the Brown-Rho scaling on nuclear matter, neutron stars and the long life-time $\beta$-decay of ${ }^{14} C$ have been carried out 14,20

In the following we shall first describe some details for solving the Dirac radial equation with a scalar central potential $V_{s}$ (section 2). To mimic the extremly strong potential which may be present in highly compacted stellar objects, we shall consider the Dirac equation for a nucleon in super-deep scalar potentials in section 3. The resulting nucleon-anti-nucleon intruder states will be discussed. In the final section 4 we present a summary and conclusion.

In order to elucidate our calculation by a simple pedagogical example, we have repeated the calculation using a one-dimensional Woods-Saxon potential; this is included in an Appendix.

\section{Dirac radial equation with scalar potential well}

We consider the Dirac equation for a nucleon

$$
\left[c(\alpha \cdot \mathbf{p})+\beta\left(m c^{2}+V_{s}\right)+V_{v}\right] \psi=E \psi
$$

where $m$ denotes its mass, and $V_{s}$ and $V_{v}$ respectively the scalar and vector potential. Its radial equation $2 \frac{224}{24}$

with

$$
\begin{aligned}
& \frac{d g(r)}{d r}=-\frac{k}{r} g(r)+\frac{1}{\hbar c}\left[E-V_{v}(r)+V_{s}(r)+m c^{2}\right] f(r) \\
& \frac{d f(r)}{d r}=+\frac{k}{r} f(r)-\frac{1}{\hbar c}\left[E-V_{v}(r)-V_{s}(r)-m c^{2}\right] g(r)
\end{aligned}
$$

$$
k= \begin{cases}-(l+1), & j=l+1 / 2 \\ l, & j=l-1 / 2\end{cases}
$$


and

$$
\psi_{j l m}=\frac{1}{r}\left[\begin{array}{c}
i g(r) y_{l m}^{j} \\
-f(r) y_{l^{\prime} m}^{j}
\end{array}\right]
$$

where $l^{\prime}=2 j-l$. In the above, we use $m c^{2}=938 \mathrm{MeV}$ and $\hbar c=197.3 \mathrm{MeV}$ fm. We shall consider in the present work the $l=0$ and $j=1 / 2$ state (namely $\mathrm{k}=-1$ ).

To determine the bound-state eigenvalues of the above Dirac radial equations, a standard procedure is to integrate from $\mathrm{r}=0$ to $r_{\text {end }}$ in two portions: (I) from $r=0$ to $r_{\text {match }}$ obtaining wave functions $g_{I}$ and $f_{I}$, and (II) from $r_{\text {end }}$ to $r_{\text {match }}$ obtaining $g_{I I}$ and $f_{I I}$, where $0<r_{\text {match }}<r_{\text {end }}$. For an energy variable $\omega$ equal to a bound-state eigenvalue the logarithmic boundary conditions at $r_{\text {match }}$ are satisfied, namely

$$
\frac{d}{d r} \log \left(g_{I}\left(\omega, r_{\text {match }}\right)\right)=\frac{d}{d r} \log \left(g_{I I}\left(\omega, r_{\text {match }}\right)\right), \omega=E_{1}, E_{2}, \cdots,
$$

and

$$
\frac{d}{d r} \log \left(f_{I}\left(\omega, r_{\text {match }}\right)\right)=\frac{d}{d r} \log \left(f_{I I}\left(\omega, r_{\text {match }}\right)\right), \omega=E_{1}, E_{2}, \cdots .
$$

Note that the above two conditions are theoretically equivalent, but 'numerically' they are often not. High accuracy is generally needed for calculating the above quantities, and when this is not met they may give different and spurious eigenvalues.

In the present work we employ the following alternative matching condition for determining the eigenvalues, namely

$$
\frac{f_{I}\left(\omega, r_{\text {match }}\right)}{g_{I}\left(\omega, r_{\text {match }}\right)}=\frac{f_{I I}\left(\omega, r_{\text {match }}\right)}{g_{I I}\left(\omega, r_{\text {match }}\right)}
$$

This single condition is 'theoretically' equivalent to the previous two conditions, Eqs.(5) and (6). But 'numerically' we have found it being considerably more accurate and efficient. In our calculations, we shall mainly use this matching condition. We shall also use both Eqs.(5) and (6) and a visual inspection of the resulting wave functions to double check our results.

We consider a nucleon in a scalar Woods-Saxon potential well

$$
V_{s}(r)=\frac{V_{0}}{1+e^{\left(r-r_{0}\right) / \delta}}
$$

with well depth $V_{0}$. We shall use a range of $V_{0}$ values from about -50 to minus few-thousand $\mathrm{MeV}$. (Possible connection of our choices with dense stellar objects like black holes will be discussed in section 4.) We shall also consider a range of $r_{0}$ values. For convenience we shall use $\delta=0.1 \mathrm{fm}$ for 
all calculations. The values for $r_{\text {match }}$ and $r_{\text {end }}$ are dependent on $r_{0}$. For example for $r_{0}=4 \mathrm{fm}$ we use $r_{\text {end }}=6 \mathrm{fm}$ and $r_{\text {match }}=4.5 \mathrm{fm}$. For this case we have also used $r_{\text {match }}=5.0 \mathrm{fm}$, with results in very good agreement (to 4 th decimal place) with the former.

To illustrate our calcualtions, we first present some of our results with a $V_{0}=-50 \mathrm{MeV}$ potential, $r_{0}=4 \mathrm{fm}$ and $\delta$ as given above. As seen in Fig.2, the wave function of $E_{1+}$ is largely dominated by its nucleon component $g(r) / r$. But the wave function for the negative energy $E_{1-}$ is dominated by its anti-nucleon component $f(r) / r$ as seen in Fig. 3. In Fig. 4 we consider the wave functions corresponding of the $E_{2+}$ state of the same potential. They are still dominated by $g(r) / r$ but have a slightly larger $f(r) / r$ component than $E_{1+}$. Note both of the wave functions in Fig. 4 have a node. Our scheme for ordering the positive energy states is $E_{1+}<$ $E_{2+}<E_{3+}<\cdots$ and $\cdots<E_{3-}<E_{2-}<E_{1-}$ for the negative energy ones.

These wave functions and those to be displayed later are normalized without the angular integration factor $4 \pi$, namely

$$
\int\left(f(r)^{2}+g(r)^{2}\right) d r=1
$$

The above optical potential is similar to the empirical optical potentials used for a nucleon in a nucleus of mass number $A \simeq 40 \stackrel{2122]}{\text { Our results }}$ indicate that for such ordinary nuclear systems the positive energy states of their nucleons are dominated by their nucleon component $g(r)$.

\section{Nucleon anti-nucleon level crossing and intruder state}

In Table 1, we present our results for a Dirac nucleon in the above potential well with different well depths $V_{0}$. Before discussing our results, let us mention that the matching conditions addressed earlier depends very sensitively on the energy variable $\omega$. Thus, a very small change in $\omega$ can drastically change the wave functions $f$ and $g$. As shown in Table 1, we have determined the energies $E$ very accurately so as to satisfy the matching conditions. It is our hope that interested readers may check our numerical results.

Let us now discuss the results listed in Table 1. In Fig. 5 we plot the energies of of the $1-, 1+$ and $2+$ states versus $V_{0}$. As seen there is clearly a level-crossing behavior; the nucleon level $E_{1+}$ descends with $V_{0}$ whereas the anti-nucleon $E_{1-}$ level ascends. They 'cross' at a crossing potential of $V_{0} \simeq-1000 \mathrm{MeV} \simeq-m c^{2}$. (In fact they can not actually cross for real 


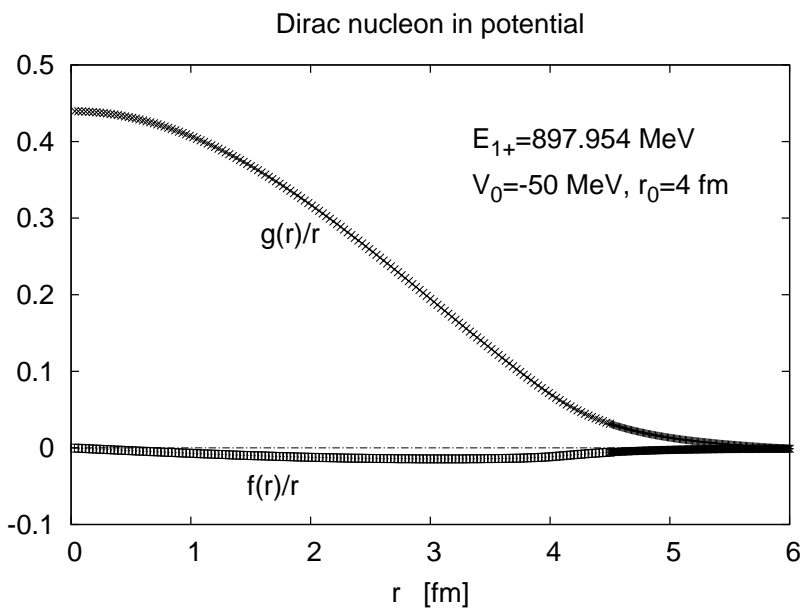

Fig. 2. Dirac wave functions for $E_{1+}=897.952 \mathrm{MeV}$ and $V_{0}=-50 \mathrm{MeV}$.

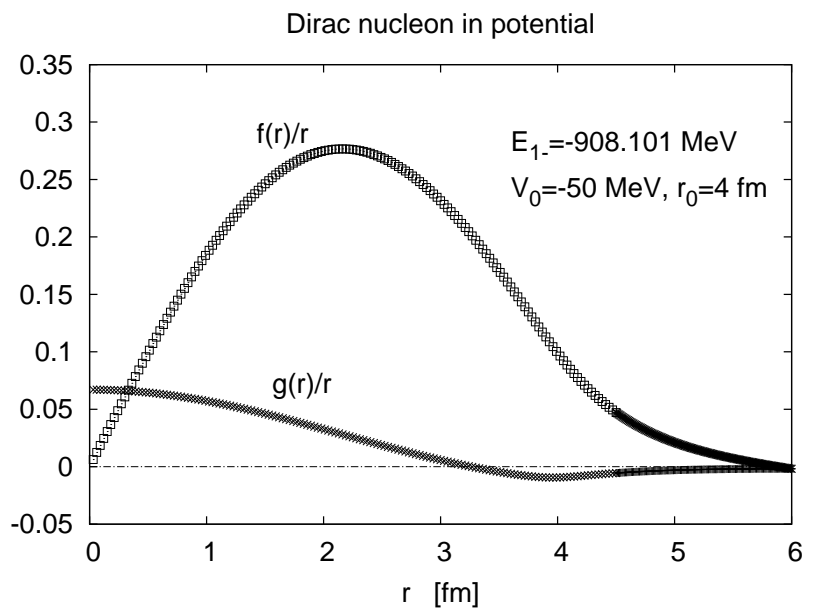

Fig. 3. Same as Fig. 2 but for $E_{1-}=-908.100 \mathrm{MeV}$.

$V_{0}$, but can do so for complex $V_{0} \cdot 9$ ) In Fig.6, we plot the corresponding anti-nucleon fractions $\langle f \mid f\rangle$. As the well becomes deeper, the anti-nucleon fraction of the 1 - state drops monotonically from $\sim 1$ at shallow well to $\sim 0$ at deep well of $V_{0} \simeq-2000 \mathrm{MeV}$. In other words, this anti-nucleon is 'transmuted' into a nucleon in the process. We note that the crossing 


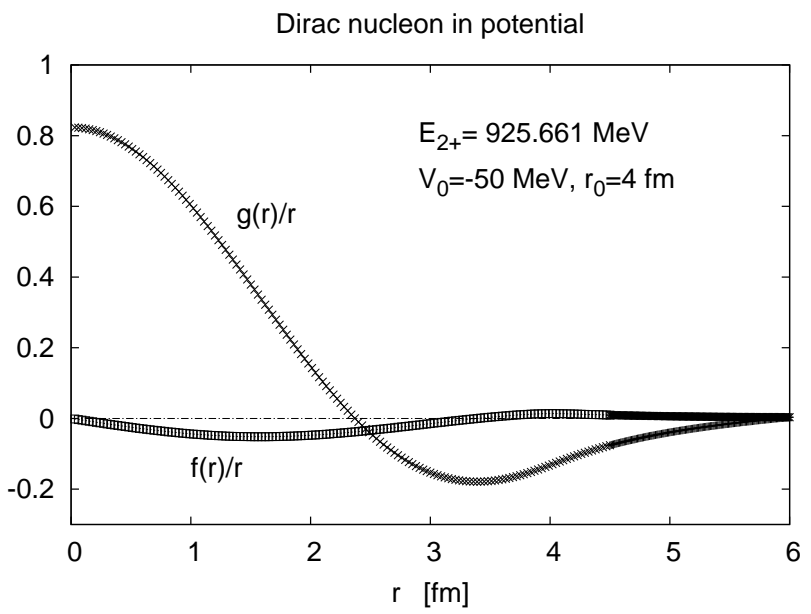

Fig. 4. Same as Fig. 2 but for $E_{2+}=925.661 \mathrm{MeV}$.

Table 1. Energies and anti-nucleon fraction $\langle f \mid f\rangle$ of a nucleon in scalar Woods-Saxon potentials of depth $V_{0}$. The widths of these potentials are all $r_{0}=4 \mathrm{fm}$. The subscripts $1+$ and $1-$ refer to respectively the lowest positive- and highest negative-energy state. $V_{0}$ and $E$ are both in units of $\mathrm{MeV}$.

\begin{tabular}{ccccc}
\hline$V_{0}$ & $E_{1-}$ & $\langle f \mid f\rangle_{1-}$ & $E_{1+}$ & $\langle f \mid f\rangle_{1+}$ \\
\hline-50 & -908.101 & 0.9908 & 897.954 & 0.004645 \\
-100 & -861.325 & 0.9883 & 849.499 & 0.005851 \\
-300 & -671.036 & 0.9780 & 654.283 & 0.01098 \\
-500 & -485.047 & 0.9570 & 461.309 & 0.02180 \\
-700 & -313.559 & 0.8594 & 275.994 & 0.05498 \\
-900 & -194.461 & 0.6487 & 120.856 & 0.2011 \\
-1000 & -191.321 & 0.3894 & 76.8122 & 0.3537 \\
-1100 & -236.603 & 0.1854 & 59.8212 & 0.4574 \\
-1300 & -397.210 & 0.05180 & 52.5618 & 0.4917 \\
-1500 & -583.955 & 0.02299 & 50.7829 & 0.4960 \\
-1700 & -777.233 & 0.01515 & 49.9197 & 0.4973 \\
\hline
\end{tabular}

potentials for $E$ and $\langle f \mid f\rangle$ are approximately the same.

We now consider the evolution of the energy and anti-nucleon fraction of the $1+$ state in Figs. 5 and 6 . As the well becomes deeper, its energy drops till about $V_{0} \simeq-1000 \mathrm{MeV}$. And until this strength is reached, this anti-nucleon fraction keeps on rising. But afterwards a plateau of energy 


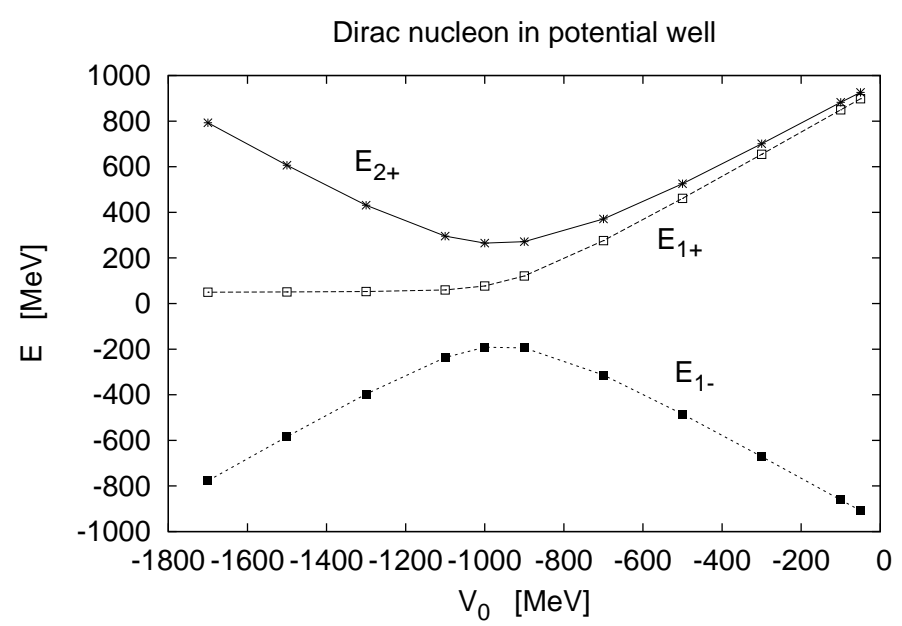

Fig. 5. Energy levels of nucleon in potential wells of different well depths $V_{0}$.

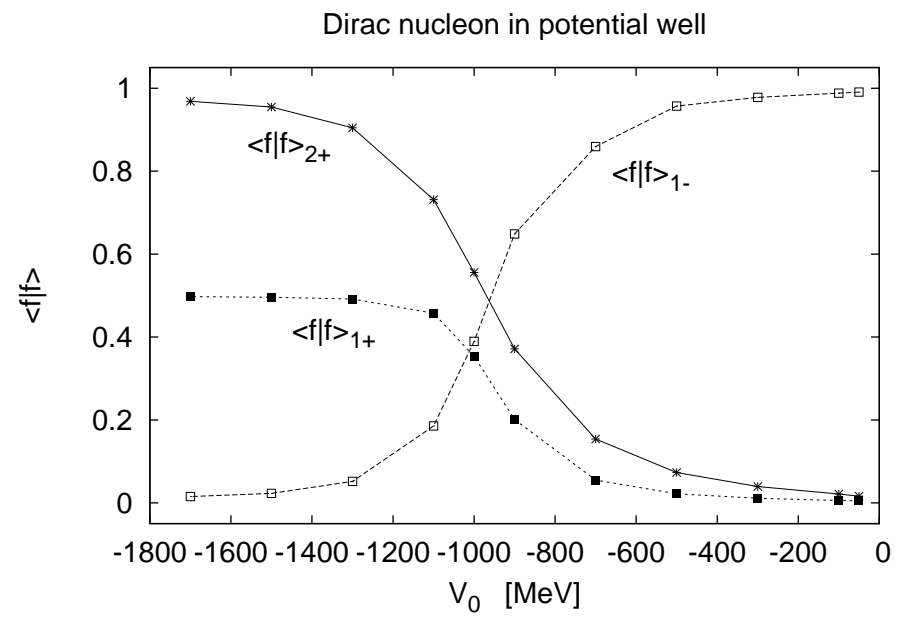

Fig. 6. Anti-nucleon fractions of nucleon in potential wells of different well depths $V_{0}$.

$\simeq 50 \mathrm{MeV}$ and $\langle f \mid f\rangle \simeq 0.50$ is reached. It is of interest that this nucleon is approaching some sort of 'hybrid nucleon' composed of half nucleon and half anti-nucleon. It may be noted that, as indicated by Fig. 5, this hybrid state is the only bound state for $V_{0}<\sim-2000 \mathrm{MeV}$, while all other states 
Dirac nucleon in potential

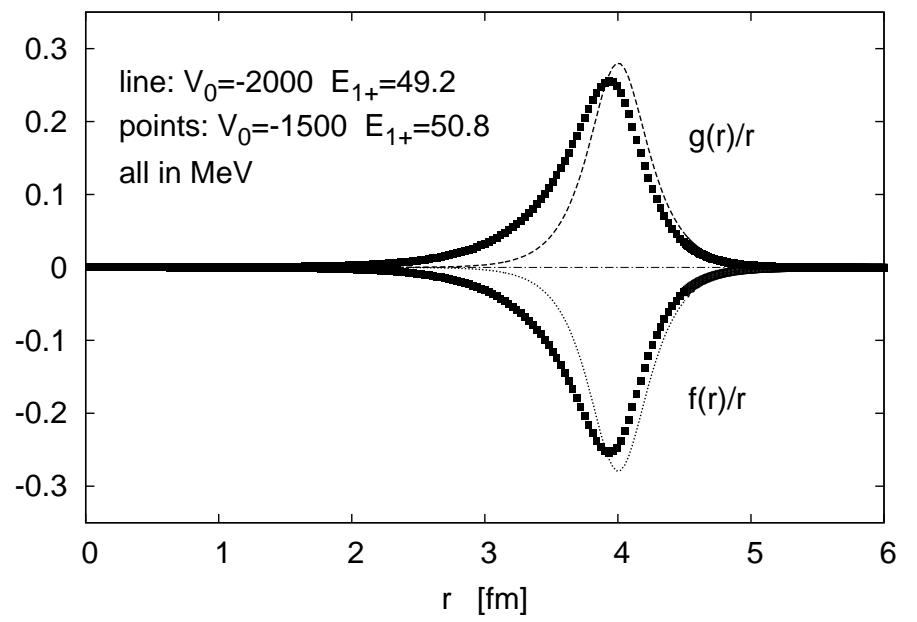

Fig. 7. Wave functions of a Dirac nucleon in a super-deep potential well of depth $V_{0}=$ $-1500 \mathrm{MeV}$ with energy $E_{1+}=50.7829 \mathrm{MeV}$. Those of the corresponding state for $V_{0}=$ $-2000 \mathrm{MeV}$ are also shown.

are diverging outward having energies $|E|>m c^{2}$. (By bound state we mean a state of energy being $|E|<m c^{2}$.)

In Fig. 7 we display the wave function of such a hybrid nucleon belonging to the $V_{0}=-1500 \mathrm{MeV}$ potential. It is amusing that the radial distributions of its nucleon and anti-nucleon components are almost identical, except for a sign change in the wave function. Furthermore, this hybrid nucleon has the mass largely concentrated near its surface, making it a hollow nucleon. In this figure, the corresponding wave function for $V_{0}=-2000$ $\mathrm{MeV}$ is also shown. It has a similar Gaussian wave packet shape, but of narrower width.

The wave function of the 1 - anti-nuleon belonging to the same -1500 $\mathrm{MeV}$ potential is displayed in Fig. 8. As seen, it has almost no anti-nucleon content; it is a 'nucleon' evolved from its parent anti-nucleon. The wave function of the $2+$ state of this potential is shown in Fig. 9. Its $f(r)$ component is much larger than the $g(r)$ one, indicating this positive energy nucleon is actualy predominantly composed of anti-nucleon.

Let us now discuss the results from potentials deeper than those shown in Table 1. In Fig. 10 we focus on the $1+$ state calculated with different well depths. Plotted are the anti-ncleon fraction $\langle f \mid f\rangle$ and the effective 


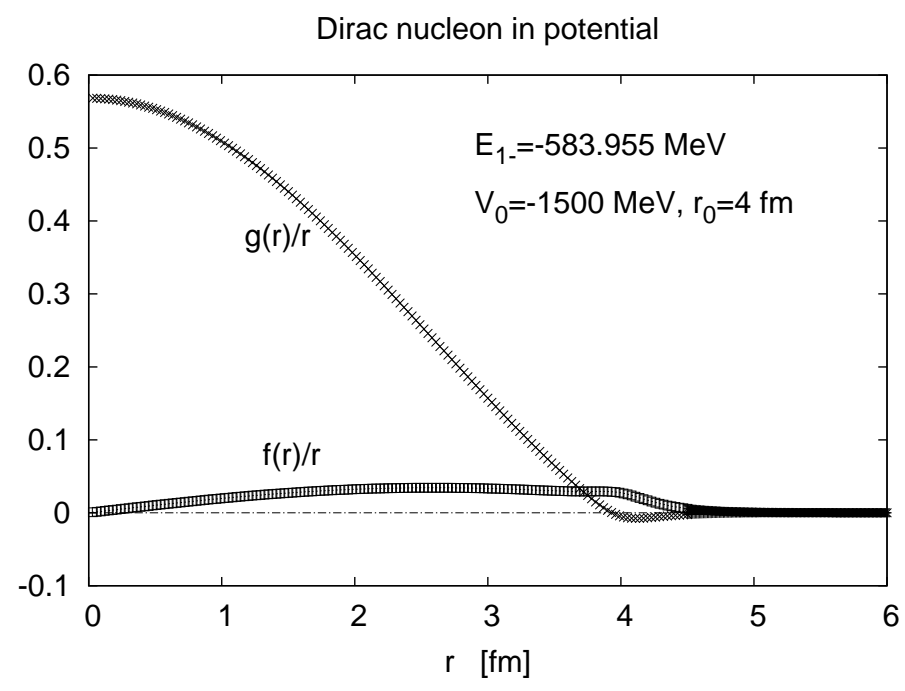

Fig. 8. Same as Fig.7 but for energy $E_{1-}=-583.955 \mathrm{MeV}$.

mass defined as $m^{*} / m \equiv E_{1+} /\left(m c^{2}\right)$ where $m$ is the free nucleon mass. Near zero potential, we have $m^{*} / m \simeq 1$ and $\langle f \mid f\rangle \simeq 0$. As $V_{0}$ becomes more negative, the former descends and the latter ascends until $V_{0} \simeq-1500$ $\mathrm{MeV}$. Afterwards, its $m * / m$ remains flat at about 0.05 (corresponding to $E_{1+} \simeq 50 \mathrm{MeV}$ ) and its $\langle f \mid f\rangle$ is flat at about 0.50 . In the figure we show results down to $V_{0} \simeq-3500 \mathrm{MeV}$. We have tried deeper potentials, in fact as deep as $V_{0}=-5400 \mathrm{MeV}$, and the flatness of these two curves remains unchanged. It seems that the above $E_{1+}$ and $<f \mid f>_{1+}$ are practically independent of $V_{0}$ for $V_{0}<\sim-1500 \mathrm{MeV}$. The wave function and energy of this 'flat' state will be referred to as $\Phi_{1 / 2}$ and $E_{1 / 2}$ respectively.

Our results presented above are all obtained numerically. It would be nice if they can be obtained analytically. We have tried but so far have not succeeded. To partially understand the above 'flatness' behavior of Figs. 5, 6 and 10, let us rewrite Eq.(2) in matrix form as

$$
\left[\begin{array}{cc}
-\left(V_{s}+m c^{2}\right) / \hbar c & \frac{d}{d r}+\frac{k}{r} \\
-\frac{d}{d r}+\frac{k}{r} & \left(V_{s}+m c^{2}\right) / \hbar c
\end{array}\right]\left[\begin{array}{l}
f \\
g
\end{array}\right]=E\left[\begin{array}{l}
f \\
g
\end{array}\right]
$$

Here we do not have the $V_{v}$ term of Eq.(2) as we include only the scalar potential $V_{s}$.

The diagonal terms $-\left(V_{s}+m c^{2}\right)$ and $\left(V_{s}+m c^{2}\right)$ play an important role in the above equation. When $V_{s} \simeq 0$, the wave functions of the positive- 


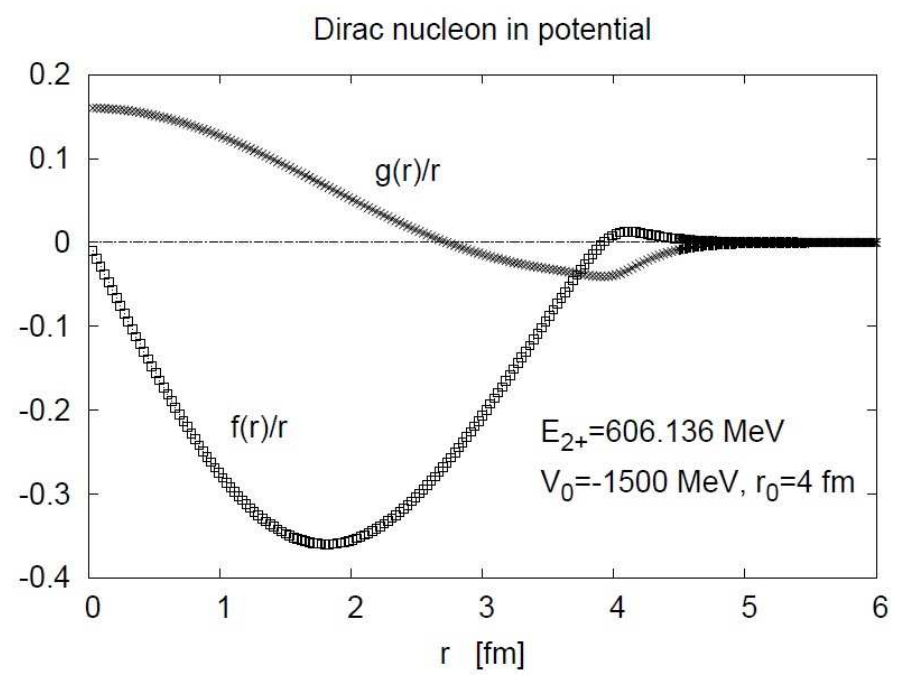

Fig. 9. Same as Fig.7 but for energy $E_{2+}=606.136 \mathrm{MeV}$.

and negative-energy states are dominated by their nucleon and anti-nucleon component $g(r)$ and $f(r)$ respectively. But when $V_{s}$ is largely negative, say $-2000 \mathrm{MeV},<f\left|-\left(V_{s}+m c^{2}\right)\right| f>$ becomes postive while $<g\left|\left(V_{s}+m c^{2}\right)\right| g>$ becomes negative. Then the positive-energy state is dominated by the anti-nucleon component $f(r)$ while the negative-energy one by $g(r)$. This explains the level crossing seen in Fig. 5 for the $1-$ to $2+$ right-to-left cross over and similarly the $2+$ to $1-$ cross over.

The middle $1+$ state of Fig. 5 is, however, a special case. Its energy $E_{1+}$ for $V_{0}<\sim-1500 \mathrm{MeV}$ is not only nearly independent of $V_{0}$ but is also rather small $(\sim 50 \mathrm{MeV})$. These properties suggest that there must be some cancellation among the contributions from $V_{s}$ to this state, leading to a near-zero net contribution. A possible way to attain such cancellation is to have either $f=-g$ or $f=g$ where $f$ and $g$ are respectively the anti-nucleon and nucleon component of the eigenfunction of this $1+$ state.

To study this possible cancellation, we rewrite Eq.(10) as

and

$$
\left[-\left(V_{s}+m c^{2}\right) /(\hbar c)+\frac{d}{d r}\right](f+g)-\frac{k}{r}(f-g)=E(f-g)
$$

$$
\left[-\left(V_{s}+m c^{2}\right) /(\hbar c)-\frac{d}{d r}\right](f-g)+\frac{k}{r}(f+g)=E(f+g) .
$$

We have used the above equations to calculate the wave functions $(\mathrm{f}+\mathrm{g})$ and (f-g). These calculations can also be performed equivalently using 


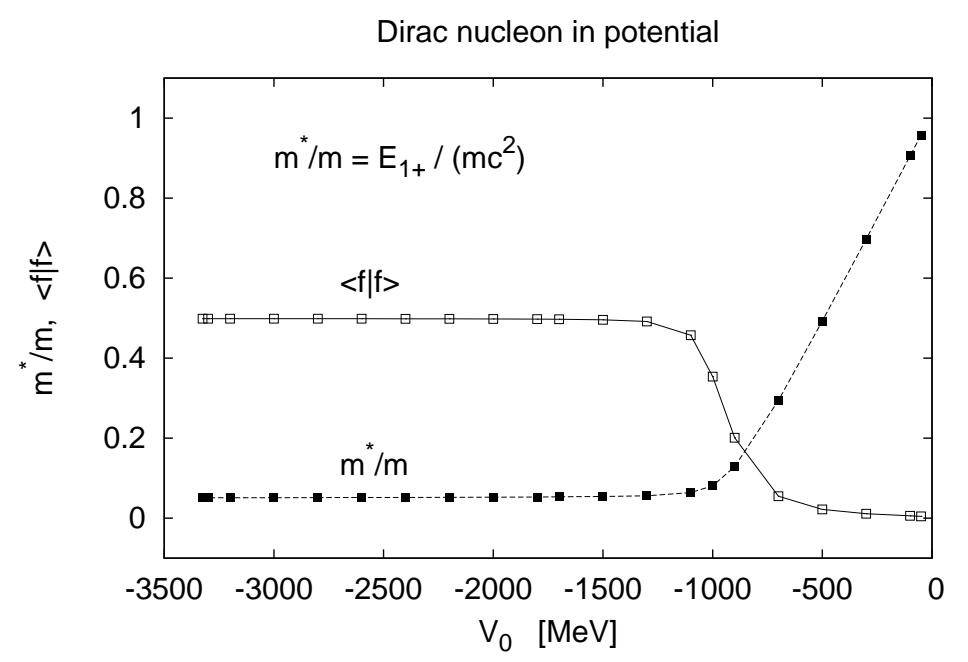

Fig. 10. Evolution of the $E_{1+}$ energies and their anti-nucleon fractions with potential depth $V_{0}$. A potential radius of $r_{0}=4 \mathrm{fm}$ is used for all calculations shown.

Eq.(10). We have found for $V_{0} \lesssim-1400 \mathrm{MeV}\left(1.5 m c^{2}=1407 \mathrm{MeV}\right)$, the wave function $(\mathrm{f}+\mathrm{g})$ is very much smaller than $(\mathrm{f}-\mathrm{g})$ for the $1+$ state. We have calculated for this state the norm $N_{+} \equiv<f+g \mid f+g>$ and $N_{-} \equiv<f-g \mid f-g>$ with the normalization $N_{+}+N_{-}=1$. Our results are $\left(N_{+}, N_{-}\right)=(0.00004,0.99996)$ for $V_{0}=-1400 \mathrm{MeV}$ and $(0.00002$, $0.99998)$ for $V_{0}=-1500 \mathrm{MeV}$. In both calculations $r_{0}=4 \mathrm{fm}$ is used.

Thus for the $1+$ state with $V_{0} \lesssim-1500 \mathrm{MeV}$, we can drop the $(f+g)$ terms relative to the $(f-g)$ ones in the above equations. Then Eq. (11) gives the simple energy relation

$$
E=-k \hbar c \frac{<g|1 / r| g>}{<g \mid g>}
$$

for the energy of the $1+$ state. Recall that $k=-1$ (see section 2). To calculate the above energy, we need to have $\langle g|1 / r| g\rangle$.

If $\langle g|1 / r| g>/<g| g>$ is independent of $V_{0}$, then so is the above energy. In fact we have found that for $V_{0} \lesssim-1500 \mathrm{MeV},(<g|1 / r| g>/<$ $g \mid g>$ ) is closely equal to $1 / r_{0}$ and is nearly independent of $V_{0}$. Thus the above energy is only weakly dependent on $V_{0}$ as indicated by Fig. 10 and Table 1 . For the above $V_{0}$ range, $g$ is generally of a Gaussian wave packet shape narrowly peaked around $r_{0}$. In Fig. 7 we have displayed such wave functions for $r_{0}=4 \mathrm{fm}$. We have found that the wave functions for other 


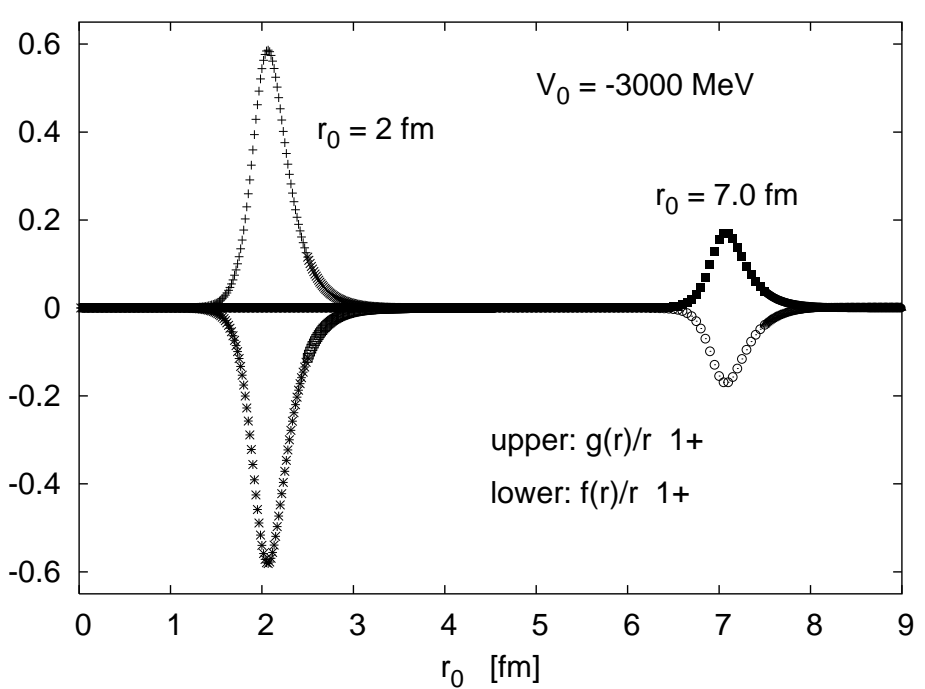

Fig. 11. Radial wave functions $g(r) / r$ and $f(r) / r$ for the $1+$ state of potential wells $V_{0}=-3000 \mathrm{MeV}$ and $r_{0}=2$ and $7 \mathrm{fm}$.

choices of $r_{0}$ are also of this shape. In Fig. 11 we display two such wave functions for $r_{0}=2$ and $7 \mathrm{fm}$. This special shape of the wave functions renders $(<g|1 / r| g>/<g \mid g>) \simeq 1 / r_{0}$ a good approximation. With this approximation, Eq. (13) becomes then

$$
E \simeq \frac{-k \hbar c}{r_{0}}
$$

The accuracy of this approximation will be discussed later.

We have considered Eq. (11) for $f \simeq-g$. Let us now consider Eq. (12). In this situation we can drop the $(\mathrm{f}+\mathrm{g})$ terms there and have

$$
\frac{d g}{d r}=-g\left(V_{s}+m c^{2}\right) /(\hbar c)
$$

which is a simple differential equation for determining the wave function $g$. For the Woods-Saxon potential of Eq.(8), the above equation gives

$$
g(r)=\exp \left[-\frac{V_{0}}{\hbar c}\left[\left(r-r_{0}\right)-\delta \log \left(1+e^{\left(r-r_{0}\right) / \delta}\right)\right]-\frac{m c^{2}}{\hbar c} r\right] .
$$

Note that the above expression does not include the normalization constant. For $V_{0} \lesssim-1500 \mathrm{MeV}$ the wave function calculated with the above equation agrees very well with the corresponding result from solving the full Dirac equation of Eq. (10). 


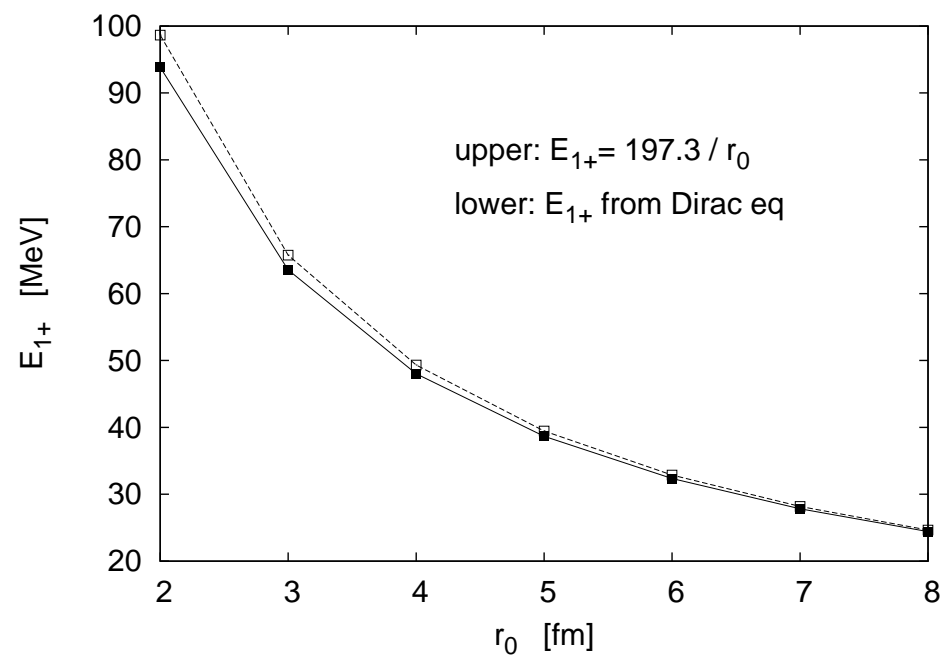

Fig. 12. Dependence of the $1+$ energy on the range $r_{0}$ of the WS potential well. Common well depth of $V_{0}=-3000 \mathrm{MeV}$ is used.

The following approximation is helpful in understanding the structure of the $g(r)$ wave function. For $r$ close to $r_{0}$, the Woods-Saxon potential of Eq.(8) can be approximated by a linear potential, namely

$$
V_{s}(r) \rightarrow \frac{V_{0}}{2}\left[1-\frac{r-r_{0}}{2 \mu}\right] .
$$

Then Eq.(15) gives

$$
g(r) \propto \exp \left[\frac{V_{0}}{8 \mu}\left(r-r_{0}\right)^{2}\right]
$$

which is of Gaussian form, providing an explanation for the shape of the wave functions displayed in Figs. 7 and 11.

The parameter $\mu$ of Eq.(17) is determined by fitting the WS potential near $r_{0}$. As an example, we find $\mu=0.144$ by fitting the $r_{0}=4 \mathrm{fm}$ and $V_{0}=-2000 \mathrm{MeV}$ potential in the range of $r=3.7$ to $4.3 \mathrm{fm}$. Then the wave function given by Eq.(18) is of the form $\exp \left[b\left(r-r_{0}\right)^{2}\right]$ with $b=V_{0} /(\hbar c 8 \mu)=-8.80$. The same wave function obtained by solving the Dirac equations of Eq. (10) has been given in Fig. 7. Fitting it by $\alpha$. $\exp \left[\beta\left(r-\gamma r_{0}\right)^{2}\right]$ gives $\alpha=1.07, \beta=-8.37$ and $\gamma=1.004$. Note that the $\beta$ value is close to the above $b$ value.

To estimate the accuracy of the energy approximation of Eq.(14), we have compared the energies so obtained with those from the full calculation 
of Eq. (10). Some sample comparisons are given in Fig. 12 where the energies for the $1+$ state calulated from Eq.(10) using $V_{0}=-3000 \mathrm{MeV}$ and $r_{0}=2$ to $7 \mathrm{fm}$ are compared with those given by Eq.(14). It is of interest that the two sets of energies agree remarkably well, indicating this approximation being highly accurate. Note that Eq. (14) is independent of $V_{0}$. This is consistent with Fig. 10 where the energies of the $1+$ state are nearly independent of $V_{0}$ for $V_{0} \lesssim-1500 \mathrm{MeV}$.

For particles of zero rest mass, the momentum-position uncertainty principle may be written as $\Delta E \Delta r \simeq \hbar c ; E=p c$. It is of interest that the energy relation of this special state as given by Eq. (14) is similar to this uncertainty principle, suggesting that the nucleon in this special state has a near-vanishing effective mass as indicated by Fig. 10 .

\section{Summary and discussion}

In the present work we have solved the Dirac radial equation for a nucleon in a scalar Woods-Saxon potential well with depth $V_{0}$ and radius $r_{0}$. For weak potentials such as $V_{0} \simeq-50 \mathrm{MeV}$, the positive-energy states $\Psi_{+}$of this equation have energies $\simeq+m c^{2}$ and wave functions dominated by the nucleon component $g(r)$, as indicated by Figs. 2 and 6 . Similarly the negative-energy states $\Psi_{-}$have energies close to $-m c^{2}$ and wave functions dominated by the anti-nucleon component $f(r)$ as illustrated in Fig. 3.

As $V_{0}$ turns more negative (deeper), a qualitative reversal of the wave functions takes place. Namely, $\Psi_{-}$is generally becoming increasingly dominated by $g(r)$, and so is $\Psi_{+}$by $f(r)$. Examples illustrating such tendencies are displayed in Figs. 8 and 9.

The lowest positive-energy state $1_{+}$is however an exemption, being a special intruder state. As indicated by Figs. 5 and 6 , at weak potential strength this state has $E_{1+} \simeq m c^{2}$ and $<f\left|\Psi_{1+}\right\rangle^{2} \simeq 0$. But as $V_{0}$ becomes $\lesssim-1500 \mathrm{MeV}$, this state is characterized by a special wave function $\Psi_{1 / 2}$ with constant composition of $f=-g$ where $g$ and $f$ are respectively its nucleon and anti-nucleon component and both are narrowly peaked around $r_{0}$. In addition, the energy of this state approaches a constant energy $E_{1 / 2} \simeq \hbar c / r_{0}$. These behaviors have been illustrated in Figs. 5, 6 and 10. For $V_{0} \lesssim-2000 \mathrm{MeV}$, our results suggest that the above state, with wave function $\Psi_{1 / 2}$ and energy $E_{1 / 2}$, is the only bound state. It should be of interest if one could check this special intruder state experimentally. We note that a hypothetical scalar Woods-Saxon potential of very strong strength has been used in our work for calculating this and other intruder 
states. Is such a strong potential conceivable?

We have estimated that a nucleon near the surface of a medium-mass black hole (of mass $\sim 10^{3}$ solar mass and radius $\sim 10^{3} \mathrm{~km}{ }^{25 \mid 26}$ ) would experience a gravitational potential of approximately $-1400 \mathrm{MeV}\left(\cong 1.5 m c^{2}\right)$. There has been evidence for super-massive black holes such as the $4.3 \times 10^{6}$ solar-mass black hole in the Milky Way ${ }^{25 \mid 26}$ They may produce similar or stronger gravitational potential. Thus, the special nucleons indicated by this work may be present near such black holes. 9 This needs of course further study. With the presence of such strong gravitational field we may need to use a Dirac equation in curved spacetime. $\frac{28 \mid 29}{}$ whereas we have been using the Dirac equation in flat spacetime. It would indeed be of interest to obtain an estimate of the effects introduced by curved spacetime.

Let us end by quoting a paragraph written by Gerry!

"One of the authors, Gerry Brown, arrived at Princeton in early September, 1964. The next morning, as he came to the Palmer Physics Laboratory, Eugene Wigner, who just preceded him, opened the door for him (It was a real contest to get ahead of Eugene and open the door for him which very few succeeded in doing.) Eugene asked Gerry, as we went into the building, what he planned to work on. 'I plan to work out the nucleon-nucleon interaction in nuclei.' Eugene said that it would take someone cleverer than him, to which Gerry replied that they probably disagreed what it meant to 'work out'. Gerry wanted to achieve a working knowledge, sufficiently good to be able to work out problems in nuclear physics."

In fact, it is our hope that the present calculations may serve as a warmup exercise for 'working out' the Dirac equation for nucleons experiencing extremely strong scalar potential fields and its possible connection with dense stellar objects.

Acknowledgement: We thank Edward Shuryak, Ismail Zahed and Jeremy Holt for many helpful discussions. This work is supported by the US Department of Energy under contract DE-FG02-88ER4038. S. Shu is supported by the China Scholarship Council.

Appendix: In this Appendix the nucleon-anti-nucleon intruder states mentioned earlier are studied using a simpler and more transparent model. As indicated by Eq.(5), our earlier calculations are based on a model where a nucleon is placed in a spherical Woods-Saxon (WS) potential. When the depth of this potential is deeper than a certain value, the ground state of

\footnotetext{
${ }^{9}$ We have also made a similar estimate for a neutron star of mass $2.1 M{ }^{27}$ and radius $\sim 11 \mathrm{~km} ! 20$ The resulting potential is about $-270 \mathrm{MeV}$. Thus we do not expect the presence of such special nucleons near this neutron star, as indicated by Fig.10.
} 
Dirac eq $1 \mathrm{~d}$-WS potential, depth $\mathrm{V}_{0}$, walls at $+/-4 \mathrm{fm}$

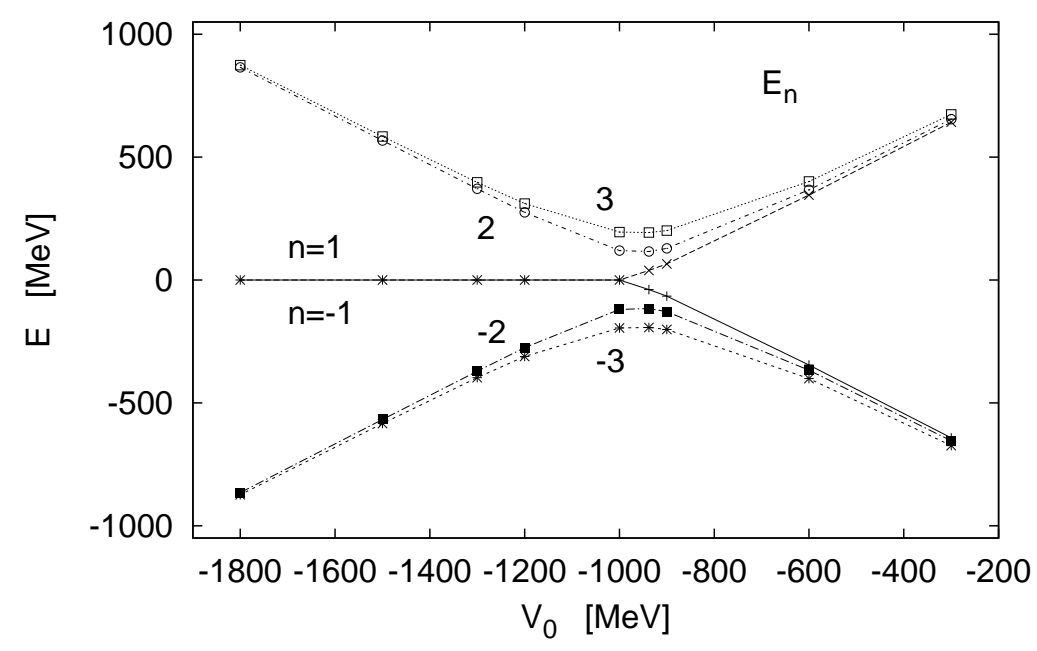

Fig. 13. Energies of Dirac equation for a nucleon in a one-dimensional scalar WS potential. A similar plot for the spherical WS potential used earlier is shown in Fig. 3.

the nucleon becomes a special half-nucleon intruder state. One might ask if this holds true also for a one-dimensional Woods-Saxon potential.

To answer this question, we have repeated our earlier calculations using a one-dimensional WS potential

$$
V_{s}(x)=V_{0}\left[1+\exp \left(\frac{x S(x)-x_{0}}{\delta}\right)\right]^{-1} ; S(x)=x /|x| .
$$

The corresponding Dirac equation is

$$
\left[\begin{array}{cc}
A & B \\
-B & -A
\end{array}\right]\left[\begin{array}{l}
f \\
g
\end{array}\right]=E\left[\begin{array}{l}
f \\
g
\end{array}\right]
$$

with

$$
A=-\left(V_{s}(x)+m c^{2}\right) /(\hbar c), \quad B=\frac{d}{d x} .
$$

The solutions of this equation are paired, namely

$$
E_{n+}=-E_{n-} ; \quad\left(f_{n+}, g_{n+}\right)= \pm\left(g_{n-}, f_{n-}\right)
$$

for all n. Eq.(20) can be rewritten as

$$
\begin{aligned}
& (A-B)(A+B)(f+g)=E^{2}(f+g) \\
& (A+B)(A-B)(f-g)=E^{2}(f-g) .
\end{aligned}
$$


Dirac eq $1 \mathrm{~d}$-WS potential, depth $\mathrm{V}_{0}$, walls at $+/-\mathrm{x}_{0}$

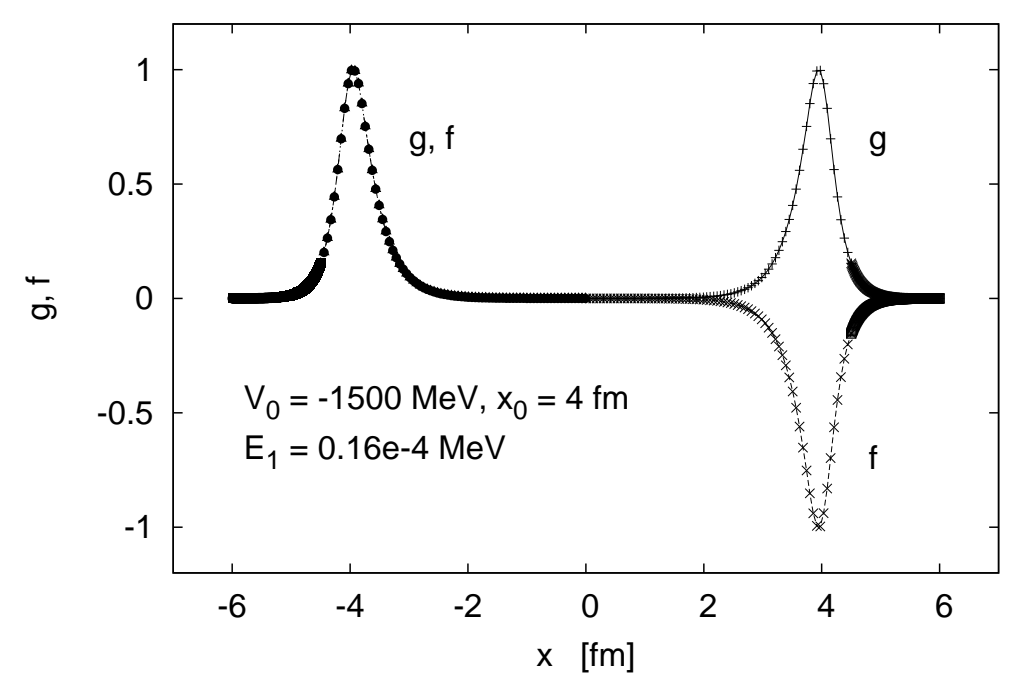

Fig. 14. Wave function of a one-dimensional half-nucleon intruder state. Similar wave functions for the spherical WS potential used earlier are shown in Figs. 7 and 11.

The energies of these equations have been calculated as illustrated in Fig. 13. (A common value of $\delta=0.1 \mathrm{fm}$ is used in all calculations.) We note that the energies are paired, in agreement with Eq. (22).

As also shown in Fig. 13, there is a clear level crossing behavior near $V_{0} \simeq-1000 \mathrm{MeV}$. For potentials shallower than this value, the wave functions for the $E_{n+}$ and $E_{n-}$ states are dominated respectively by $g$ and $f$. For potentials deeper, the energies of the $E_{1+}$ and $E_{1-}$ states are special, both being practically zero and independent of the potential depth, and their wave functions all have a special structure of either $f=-g$ or $f=g$ as illutrated in Fig. 14. Also for this potential range, the wave functions for the $\left(E_{n+}, n>1\right)$ states are dominated by $f$ as illustrated in Fig. 15. Similarly those for the $\left(E_{n-}, n>1\right)$ states are dominated by $g$.

According to Eq. (20), the wave function $(f, g)$ as a whole has a 'mixed parity' structure, in the sense that each of $f$ and $g$ is a function of definite parity but their parities are opposite. This is seen in Fig. 14 where $g$ has positive parity while $f$ has negative parity. Similar parity structure is also seen in Fig. 15. We note that Eq. (23) provides a convenient way for obtaining approximate wave functions near the walls and and near the center of the potential. This has been helpful in explaining the shapes of 


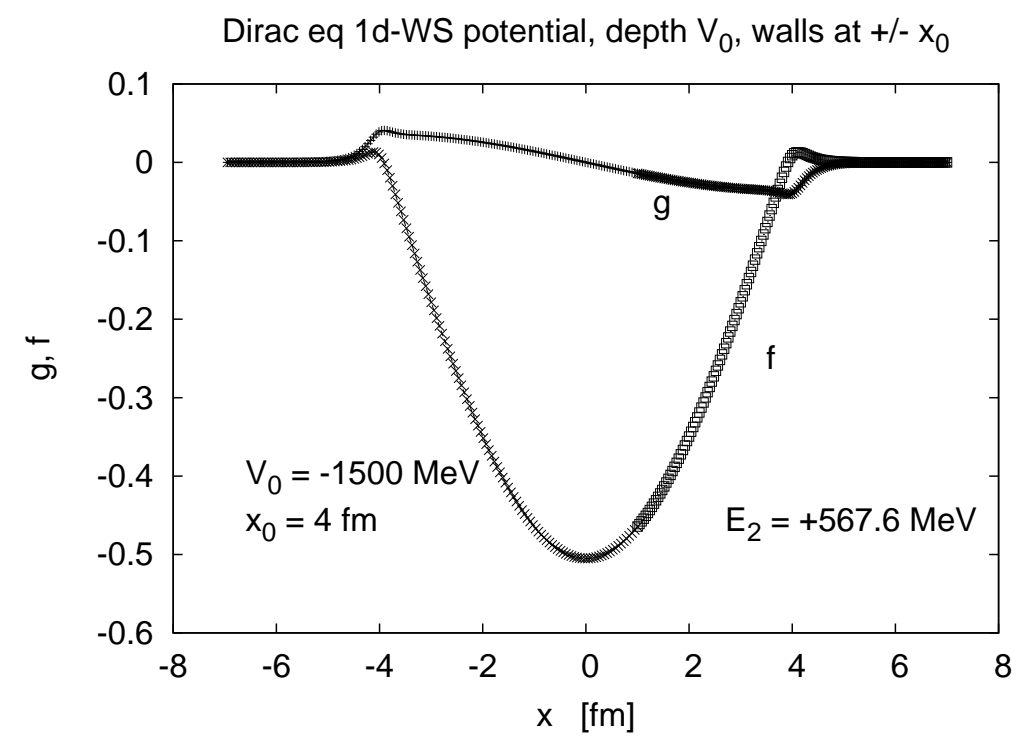

Fig. 15. Wave function of a one-dimensional positive-energy state with $f$ dominance. Similar wave functions for the spherical WS potential used earlier are shown in Fig. 9

the wave functions of Eq.(20).

In short, the main features of the nucleon-anti-nucleon intruder states obtained from the spherical WS potential are well reproduced by the results from the one-dimensional WS potential.

\section{References}

1. G.E. Brown and T.T.S. Kuo, Nucl. Phys. 92, 481 (1967).

2. T. T. S. Kuo and G. E. Brown, Nucl. Phys. 85, 40 (1966).

3. G. E. Brown, Unified Theory of Nuclear Models and Forces (North-Holland, Amsterdam, 1971).

4. M. Hjorth-Jensen, T.T.S. Kuo and E. Osnes, Physics Report 261, 126 (1995).

5. L. Coraggio, A. Covello, A. Gargano, N. Itaco and T. T. S. Kuo, Prog. Part. Nucl. Phys. 62, 135 (2009).

6. G. E. Brown, T. T. S. Kuo, J. W. Holt and S. Lee, 'Nucleon-nucleon interactions and nuclear many-body problems', World Scientific Pub. Co. Singapore (2010).

7. H. Dong, T.T.S. Kuo and J.W. Holt, Nucl. Phys. 930, 1 (2014).

8. T.T.S. Kuo, J.W. Holt and E. Osnes, Physica Scripta 91, 033009 (2016).

9. T. H. Schucan and H. A. Weidenmueller, Ann. Phys. (N.Y.) 73, 103 (1972); 76, 483 (1973). 
10. P.J. Ellis and E. Osnes, Rev. Mod. Phys. 49, 777 (1977); C. M. Vincent and S. Pittel, Phys. Lett. 47B, 327 (1973); P. A. Schaefer, Ann. Phys. 87, 375 (1974).

11. G. E. Brown and M. Bolsteri, Phys. Rev. Lett. 3, 377 (1959).

12. G. E. Brown and M. Rho, Phys. Rev. Lett. 66, 2720 (1991).

13. G. E. Brown and M. Rho, Phys. Rep. 396, 1 (2004) 253,252(1991).

14. J. W. Holt, G.E. Brown, J.D. Holt and T.T.S. Kuo ,Nucl. Phys. A785, 322 (2007).

15. J. W. Holt, G.E. Brown, T.T.S. Kuo, J.D. Holt and R. Machleidt, Phys. Rev. Lett. 100, 062501 (2008).

16. L. W. Siu, J. W. Holt, T. T. S. Kuo and G. E. Brown, Phys. Rev. C79, 0540004 (2009).

17. H. Dong, T.T.S. Kuo and R. Machleidt, Phys. Rev. C80, 065803 (2009).

18. H. Dong, T.T.S. Kuo and R. Machleidt, Phys. Rev. C 83, 054002 (2011).

19. H. Dong, T.T.S. Kuo, H. K. Lee, R. Macleidt and M. Rho, Phys. Rev. C87, $054332(2013)$.

20. W. G. Paeng, T.T.S. Kuo, H. K. Lee and M. Rho, arXiv:1508.05210 (2015); Phys. Rev. C, to be published (2016).

21. P. E. Hodgson, 'The nucleon optical potential' (World Scientific Pub. Co. 1994).

22. Y. Nogami and F.M. Toyama, Phys. Rev. C42, 2449 (1990).

23. R. H. Landau, 'Quantum Mechanics II', John Wiley \& Sons (1996).

24. R. R. Silbar and T. Goldman, Eur. J. Phys. 32, 212 (2011).

25. 'Black Hole-Wikipedia', https://en.wikipedia.org/wiki/Black_hole.

26. B. Schutz, 'General Relativity', Cambridge Univ. Press (2009).

27. J. Antoniadis et al. Science 340, 6131 (2013).

28. B. Mukhopadhyay and S. Chakrabarti, Nucl. Phys. B852, 629 (2000).

29. S. M. Carroll, 'Spacetime and Geometry', Addison and Wesley (2004). 